\title{
Association between coping mechanisms and adherence to diabetes-related self-care activities: a cross-sectional study
}

This article was published in the following Dove Press journal:

Patient Preference and Adherence

17 July 2017

Number of times this article has been viewed

\author{
Alin Albai' \\ Alexandra Sima' \\ Ion Papava² \\ Deiana Roman' \\ Bogdan Andor ${ }^{3}$ \\ Mihai Gafencu ${ }^{4}$ \\ 'Second Department of Internal \\ Medicine, ${ }^{2}$ Department of \\ Neurosciences, ${ }^{3}$ Department of \\ Orthopedics, ${ }^{4}$ Department of \\ Pediatrics, Victor Babeș University of \\ Medicine and Pharmacy, Timisoara, \\ Romania
}

\begin{abstract}
In the overall management of the most chronic diseases, including diabetes mellitus (DM), adherence to recommended disease-related self-care activities is of paramount importance. The diagnosis and presence of a chronic disease may be considered a difficult and stressful situation in life, a situation in which coping mechanisms are psychological processes developed at a conscious level to manage these situations. This study aimed to explore the possible relationship between the dominance of one of the four major coping styles and adherence to diabetesrelated self-care activities (DRSCAs) in the population of patients with type 2 DM (T2DM). In a cross-sectional consecutive-case population-based study design, 126 patients previously diagnosed with T2DM were enrolled. Coping mechanisms were evaluated using the Cope scale inventory, which identifies the dominant coping mechanism: problem-, emotion-, social support-, or avoidance-focused. The quality of DRSCA was evaluated using the summary of diabetes self-care activities questionnaire, in which a higher score was associated with improved adherence. In the study cohort, 45 patients (35.7\%) had problem-focused coping, $37(29.4 \%)$ had emotion-focused coping, 32 (25.4\%) social support-focused coping, and $12(9.5 \%)$ had avoidance-focused coping. Patients with emotion-focused coping had the highest level $(P=0.02)$ of DRSCA (median 44 points), followed by patients with social support-focused coping (median 40 points) and problem-focused coping (median 36 points), while patients with avoidancefocused coping had the lowest SDSCA total score (33 points). The type of dominant coping mechanism has a significant impact on the quality of the DRSCA measures implemented by the patient to manage their diabetes. Patients with emotion-focused and social support-focused coping styles tend to have significantly increased adherence to DRSCA scores, while patients with other dominant coping styles are less interested in managing their disease.
\end{abstract}

Keywords: coping, type 2 diabetes mellitus, diabetes self-management, glycemic control, diabetes prognosis

\section{Background and aims}

Diabetes mellitus (DM) is nowadays a major public health issue, with continuously increasing prevalence in both of its major two components: type 1 (T1DM) and T2DM. ${ }^{1,2}$ It is estimated that nowadays, 415 million adults are living with T2DM and that this number will increase to 642 million in $2040 .{ }^{1}$ The presence of DM has a major impact on both the diagnosed individual and the public health system. Patients with DM have decreased quality of life, ${ }^{3}$ mediated especially by the presence of acute and chronic DM complications, and it is known that DM is the major cause of atraumatic amputations worldwide, frequently leading (mediated by associated chronic kidney disease) to dialysis, a major cause of blindness (mediated by the presence of diabetic retinopathy). ${ }^{1}$
Correspondence: Alexandra Sima Second Department of Internal Medicine, Victor Babeș University of Medicine and Pharmacy, 2 Eftimie Murgu,

Timisoara 30004I, Romania

Tel +40 722467247

Fax +40256462856

Email alexa_moisuc@yahoo.com 
Regarding macrovascular complications, the presence of T2DM is considered an equivalent of cardiovascular disease. ${ }^{1}$ The impact on society and on public health systems of DM is emphasized by the high direct (especially related to DM treatment or hospitalization) or indirect (related to the patient's permanent/temporary incapacity to work or reduced productivity due to DM acute and chronic complications and decreased life expectancy) costs: in the US, it was estimated

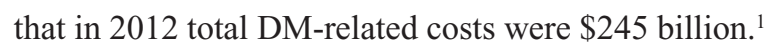

The complications of DM and thus associated disease burden may be avoided by obtaining optimal glycemic control during the patient's lifetime. It has been demonstrated that optimal DM global management may only be achieved by implementing lifestyle-optimization measures and adequate pharmacological treatment. ${ }^{4}$ As DM is a chronic, lifelong disease, paramount in its global management are diabetes-related self-care activities (DRSCAs), which in fact are all the measures taken by the patient at home to control the disease and include but are not limited to taking prescribed medication, adhering to an adequate diet, physical exercise, proactive screening of diabetes complications, and glycemic self-measurement. ${ }^{4}$

Coping is a psychological process developed at a conscious level used when one tries to manage difficult and stressful situations in life. Coping styles may be adaptive (meaning that the individual tries to reduce the stress) or maladaptive (described by a situation in which the individual keeps or even amplifies the current symptomatology). Coping has been demonstrated to be able to influence the individual's response at a biological level, leading to a normal or pathological reaction in humans, a mechanism that depends on its efficiency in reducing the psychological distress. ${ }^{5}$ Different coping mechanisms have already been demonstrated to be associated with improved or worse prognosis in other chronic diseases, such as chronic obstructive pulmonary disease. ${ }^{6}$

Our study aimed to evaluate a possible association between the dominant coping styles and the quality of the DRSCA in a cohort of patients with T2DM. Since the relationship between the quality of DRSCA and improved DM prognosis has already been demonstrated, the relationship evaluated in the present paper may thus indirectly emphasize the association between different coping mechanisms and the prognosis of DM.

\section{Patients and methods Study design and patients}

In this cross-sectional, noninterventional study, 126 patients with T2DM hospitalized in the diabetes clinic of the Pius Brinzeu Emergency Hospital (Timisoara, Romania) due to metabolic imbalances during November 2016 to March 2017 were included. Inclusion of patients was conducted according to consecutive-case population-based criteria. All patients provided written informed consent for participation in this study. The study protocol, procedures, and informed-consent template were approved by the ethics committee of Pius Brinzeu Emergency Hospital.

Inclusion criteria for this study were:

- at least 18 years old at time of inclusion

- diagnosed prior to the study with T2DM

- hospitalization for T2DM-related imbalances

- understanding of study procedures, agreement to participate, and signing of the informed-consent form.

Exclusion criteria were:

- presence of acute T2DM complications at inclusion (diabetic ketoacidosis, hyperosmolar state)

- any kind of psychiatric disorder that may interfere with study procedures and results

- inability to understand study procedures or provide consent for participation.

\section{Anthropometric, laboratory, and clinical assessments}

Data on patient age, sex, and T2DM history, including history of T2DM treatment, were collected from medical records. $\mathrm{HbA}_{1 \mathrm{c}}$, used to evaluate quality of glycemic control, was measured using an NGSP-standardized and DCCT-compliant immunoturbidimetric assay (Roche), with an intermeasurement coefficient of variation of $1.64 \%$ according to the manufacturer's specifications. As per the American Diabetes Association's 2017 standard of medical care in diabetes, in this study therapeutic goals regarding the management of T2DM were considered to be achieved in cases of $\mathrm{HbA}_{1 \mathrm{c}} \leq 7 \%{ }^{4}$

Lipid-profile measurements (total cholesterol, highdensity-lipoprotein cholesterol, low-density-lipoprotein cholesterol, and triglycerides) were performed after at least 12 hours of fasting, the serum drawn being analyzed using a standardized chemiluminescence method.

Abdominal circumference was measured three times in a row for all studied patients using a measurement tape, and the median value was recorded. The measurement was done around the abdomen at the level of the iliac crest. Since all the studied patients were Europeans of white/Caucasian race, normal values were considered to be $\leq 80 \mathrm{~cm}$ in females and $\leq 94 \mathrm{~cm}$ in males. Body mass index (BMI) was obtained by dividing the weight measured in kilograms to the square of the height measured in meters. The diagnosis of obesity was based on the BMI criteria of the World Health Organization classification: underweight $\left(<18.5 \mathrm{~kg} / \mathrm{m}^{2}\right)$, normal range $\left(18.5-24.99 \mathrm{~kg} / \mathrm{m}^{2}\right)$, overweight 
(25-29.99 kg/m²), obese class I $\left(30-34.99 \mathrm{~kg} / \mathrm{m}^{2}\right)$, obese class II (35-39.99 kg/m²), and obese class III $\left(\geq 40 \mathrm{~kg} / \mathrm{m}^{2}\right)$. Patient-weight measurements were performed in the morning of study inclusion using a calibrated weight scale, fasting, with patients wearing hospital robes or very light clothing. Diagnosis and staging of chronic kidney disease was performed according to Kidney Disease: Improving Global Outcomes 2012 guidelines, based on the estimated glomerular filtration rate, which was calculated using the Chronic Kidney Disease Epidemiology Collaboration formula. ${ }^{8}$

For the purpose of diagnosing diabetic neuropathy, trained personnel used the Michigan Neuropathy Screening Instrument (MNSI). This is able to diagnose and quantify distal symmetrical peripheral neuropathy in diabetes, and is widely used for DM as a validated score instrument. The MNSI is comprised of two parts: the examination of lower extremities, including ankle reflexes, inspection, and vibratory-sensation assessment, only abnormal findings being scored; and a self-administered questionnaire consisting of 15 items, of which only abnormal answers provided by the patient are summed. Compared to the individual DM tests performed separately, the MNSI has been shown to provide higher specificity and sensitivity. A clinical examination score of 2.5 or above or a questionnaire score of 7 or above is consistent with a positive DM diagnosis. ${ }^{9}$

Evaluation of the presence and severity of depression was done using the Patient Health Questionnaire (PHQ)-9. The nine criteria on which the diagnosis of DSM-IV depressive disorders are based can be found in the nine items forming the PHQ-9 test. The fact that it can establish the existence of depressive distress and the severity of depressive symptoms with the same nine items makes the PHQ-9 a dual-purpose instrument. Severe depression and a high PHQ-9 score are associated. Based on the obtained score, the severity of depression can be ranked as follows: severe (PHQ-9 score over 19), moderate (10-19) and minimal/mild (under 10). ${ }^{10}$

The presence and severity of anxiety disorder was assessed using the Generalized Anxiety Disorder (GAD)-7 questionnaire. This questionnaire was designed to screen and measure the severity of generalized anxiety disorder, a higher score being associated with more severe anxiety. Cutoff points for scores obtained are: 0-9 points, mild or absent anxiety; 10-19 points, moderate anxiety; and $\geq 20$, severe anxiety. ${ }^{11}$

\section{Assessment of diabetes-related self-care activity quality}

For assessment of DRSCA, the Summary of Diabetes-Related Self-Care Activities (SDSCA) questionnaire was used.
This tool is a validated questionnaire used for measurement of the quality of DRSCA performed by the patient at home. A higher SDSCA score is associated with improved selfcare of diabetes by the evaluated patients. This questionnaire includes items related but not limited to general and diabetes-specific diet measures, exercise performed daily as per doctor's recommendation, glycemic self-measurement, foot care, and smoking habits. The SDSDCA has proved to be a brief and easy to administer yet reliable and valid selfreport measure of diabetes self-management that can be used in both research and clinical practice settings. ${ }^{12}$

\section{Coping mechanisms}

Coping mechanisms were evaluated using the translated, adapted, and Romanian population-validated Cope-scale inventory, proposed by Craşovan and Sava. ${ }^{13}$ This instrument contains 60 items graded on a Likert scale of 1-4, where 1 means "I usually don't do this" and 4 means "I often do this" regarding several coping strategies used to manage stressful situations during life. The four coping styles analyzed by this instrument are:

- problem-focused coping: planning, active approach, and deletion of concurrent activities

- emotion-focused coping: positive interpretation and growth, restraint, and acceptance

- social support-focused coping: social instrumental support, use of social emotional support, and expression of feelings

- avoidant-focused coping: denial and mental and behavioral deactivation.

The highest score recorded in these four coping styles was considered in this study to be the dominant and most representative for the patient investigated.

\section{Statistical analysis}

Data were collected and analyzed using SPSS version 17 (SPSS Inc, Chicago, IL, USA), and are presented as means \pm standard deviation for continuous variables with Gaussian distribution, medians and interquartile range for continuous variables without Gaussian distribution, or percentages for categorical variables. To assess the significance of the differences between groups, Student's $t$-test or analysis of variance (means, Gaussian populations), Mann-Whitney $U$ test, or Kruskal-Wallis (medians, non-Gaussian populations) and $\chi^{2}$ (proportions) tests were used. Continuous-variable distributions were tested for normality using the Shapiro-Wilk test, and for equality of variance using Levene's test. The strength of association between two continuous variables from non-Gaussian populations was evaluated using 
Spearman's correlation coefficient. Sample-size calculation was performed prior to the study, aiming to provide a confidence level of $95 \%$ and a statistical power of at least $80 \%$. In this study, $P<0.05$ was considered the threshold for statistical significance.

\section{Results}

\section{Baseline characteristics}

The cohort studied comprised 126 patients previously diagnosed with T2DM: 70 men (55.6\%) and 56 women $(44.4 \%)$. The median age in this group was 59 years, and median diabetes duration 11.5 years. In this group of patients, $23(18.3 \%)$ achieved the glycemic control goal of $\mathrm{HbA}_{1 \mathrm{c}} \leq 7 \%$. In this group, 34 patients $(27 \%)$ were overweight and 82 (65.1\%) obese. Patient baseline characteristics are presented in Table 1.

\section{Coping mechanisms and diabetes-related self-care activities}

In the study cohort, $45(35.7 \%)$ had the problem-focused coping type, 37 (29.4\%) emotion-focused, 32 (25.4\%) social support-focused, and 12 (9.5\%) avoidance-focused. Median scores for the four coping scales stratified according to the dominant coping style are presented in Table 2.

When analyzing the coping-score distributions, we observed that patients with both dominant problem-focused (median 41 points) and emotion-focused (median 38 points) styles had the second- and third-highest scores on the social

Table I Patient baseline characteristics

\begin{tabular}{|c|c|}
\hline Parameter & Value \\
\hline Male sex (\%) ${ }^{\mathrm{a}}$ & $70(55.6)$ \\
\hline Age, years ${ }^{\mathrm{b}}$ & $59(16)$ \\
\hline Diabetes duration, years ${ }^{b}$ & II.5 (9) \\
\hline $\mathrm{HbA}_{\mathrm{Ic}}(\%)^{\mathrm{c}}$ & $8.4 \pm 1.6$ \\
\hline $\mathrm{BMI}\left(\mathrm{kg} / \mathrm{m}^{2}\right)^{\mathrm{c}}$ & $31.7 \pm 5.1$ \\
\hline Neuropathy (\%) ${ }^{\mathrm{a}}$ & $87(69)$ \\
\hline Chronic kidney disease (\%) ${ }^{\mathrm{a}}$ & $37(29.4)$ \\
\hline Uncontrolled hypertension (\%) & $42(33.3)$ \\
\hline Dyslipidemia $(\%)^{\mathrm{a}}$ & $83(65.9)$ \\
\hline Generalized anxiety disorder (\%) & $25(19.8)$ \\
\hline Depression disorder (\%) & II (8.7) \\
\hline Treatment with metformin (\%) & $71(56.3)$ \\
\hline Treatment with sulfonylurea (\%) & $9(7.1)$ \\
\hline Treatment with DPP4i (\%) & $19(15.1)$ \\
\hline Treatment with GLPI RA (\%) & $3(2.4)$ \\
\hline Treatment with basal insulin (\%) & $82(65.1)$ \\
\hline Treatment with rapid-acting insulin (\%) & $73(57.9)$ \\
\hline
\end{tabular}

Notes: ${ }^{a}$ Dichotomous variables. Results presented as $\mathrm{n}$ (\%). ' Numeric variables without Gaussian distribution. Results presented as median and interquartile range. 'Numeric variables with Gaussian distribution. Results presented as mean \pm standard deviation.

Abbreviations: BMI, body mass index; DPP4i, DPP4i inhibitor; RA, receptor agonist.
Table 2 Median coping-scale score according to dominant coping style

\begin{tabular}{lllll}
\hline Coping scales & $\begin{array}{l}\text { Problem } \\
(\mathbf{n}=\mathbf{4 5})\end{array}$ & $\begin{array}{l}\text { Emotion } \\
(\mathbf{n}=\mathbf{3 7})\end{array}$ & $\begin{array}{l}\text { Social support } \\
(\mathbf{n}=\mathbf{3 2})\end{array}$ & $\begin{array}{l}\text { Avoidance } \\
(\mathbf{n}=\mathbf{I 2})\end{array}$ \\
\hline Problem scale & $4 \mathrm{I}(\mathrm{II})$ & $32(8)$ & $37(\mathrm{I})$ & $32(\mathrm{II})$ \\
Emotion scale & $30(\mathrm{I})$ & $38(8)$ & $33(15)$ & $3 \mathrm{I}(8)$ \\
Social support & $28(\mathrm{I})$ & $30(8)$ & $44(12)$ & $35(\mathrm{I})$ \\
$\begin{array}{l}\text { scale } \\
\text { Avoidance scale }\end{array}$ & $26(\mathrm{II})$ & $28(10)$ & $27(14)$ & $40(9)$ \\
\hline
\end{tabular}

Notes: Variables with non-Gaussian distributions. Values presented median and interquartile range.

support-focused scale ( 37 and 33 points, respectively) and patients with dominant social support-focused style (median 44 points) had the second-highest score on the avoidance-focused scale (median 35 points), while patients with dominant avoidance-focused coping (median 40 points) had lower and similar scores for the other scales (median 26-28 points).

The results revealed that patients with diabetes and emotion-focused coping style had the highest level of DRSCA, with a median total SDSCA score of 44 points, followed by patients with social support-focused coping (median total SDSCA score of 40 points), problem-focused coping (median total SDSCA 36 points), while patients with avoidance-focused coping had the lowest SDSCA total score (33 points). The variances observed for these scores among the four groups, stratified in respect to the dominant coping style, were statistically significant $(P=0.02$, Kruskal-Wallis test, Figure 1). The coping styles proved to have no significant

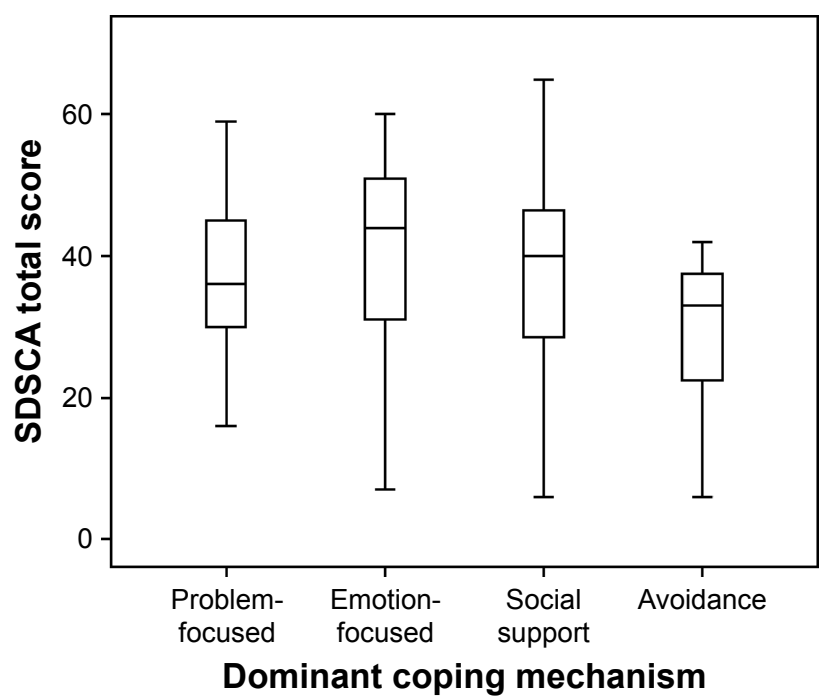

Figure I SDSCA total score according to dominant coping style. Notes: Data presented as box plots. Central lines represent median values, boxes interquartile range, and whiskers minimum and maximum values in subgroup. Abbreviation: SDSCA, diabetes-related self-care activity. 
Table 3 SDSCA scores stratified in respect to the dominant coping style

\begin{tabular}{|c|c|c|c|c|c|}
\hline SDSCA score & $\begin{array}{l}\text { Problem } \\
(n=45)\end{array}$ & $\begin{array}{l}\text { Emotion } \\
(n=37)\end{array}$ & $\begin{array}{l}\text { Social support } \\
(n=32)\end{array}$ & $\begin{array}{l}\text { Avoidance } \\
(n=12)\end{array}$ & $P$-value \\
\hline Total score & $36(16)$ & $44(22)$ & $40(19)$ & $33(17)$ & $0.02 *$ \\
\hline Diet score & $13(9)$ & $14(4)$ & $13(8)$ & $12(9)$ & 0.249 \\
\hline Exercises & $7(8)$ & $12(7)$ & $10(11)$ & $5(8)$ & $0.007^{*}$ \\
\hline Glycemic measurements & $10(10)$ & $12(6)$ & $12(6)$ & $9(12)$ & 0.406 \\
\hline Foot care & $6(12)$ & $8(\mathrm{II})$ & $6(8)$ & $3(I I)$ & 0.542 \\
\hline
\end{tabular}

Notes: ${ }^{*} \alpha<0.05$. Variables with non-Gaussian distributions. Values presented as median and interquartile range. $P$-values calculated using Kruskal-Wallis test. Abbreviation: SDSCA, diabetes-related self-care activity.

impact on adherence to diet, glycemic self-measurement, or foot-care scores (Table 3).

As for the SDSCA subscales, only on the exercise scale did we observe significant differences among the four subgroups ( $P=0.007$, Kruskal-Wallis test). In this case also, patients with emotion-focused coping (median SDSCA exercise score 12 points) were the most adherent to the physical exercises interventions proposed in contrast to patients with avoidance-focused coping (median SDSCA exercise score 5 points, Figure 2).

We observed significant associations between coping styles and several diabetes-related parameters (Table 4). Patients with emotion-focused coping were more likely to obtain optimal glycemic control, defined as $\mathrm{HbA}_{1 \mathrm{c}}$ $<7 \%$ ( $29.7 \%$ of patients), in contrast to patients who used social support-focused coping, a group in which no patient obtained optimal glycemic control $(P=0.012)$. The prevalence of dyslipidemia showed important variations in prevalence $(P=0.017)$, the highest being in the group of

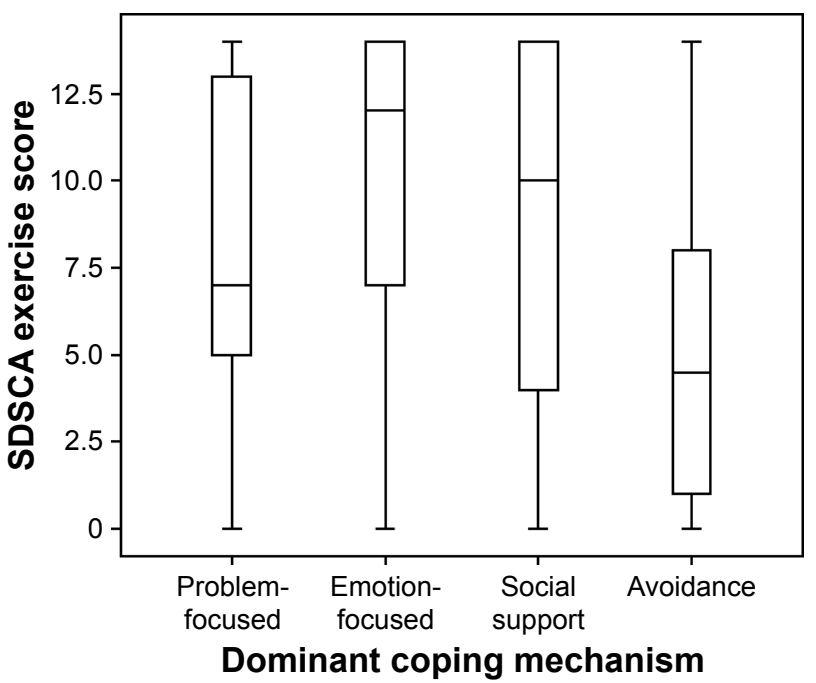

Figure 2 SDSCA exercise score according to dominant coping style. Notes: Data presented as box plots. Central lines represent median values, boxes interquartile ranges, and whiskers minimum and maximum values in subgroup. Abbreviation: SDSCA, diabetes-related self-care activity. patients with dominant problem-focused coping $(82.2 \%)$ and the lowest corresponding to patients with avoidancefocused coping (50\%). Patients with problem-focused and social support-focused coping had more pronounced neuropathy symptomatology (MNSI scores 11.5 and 10 points, respectively) compared to patients with avoidance-focused and emotion-focused coping (8.5 and 6.5 points, respectively). The variances in MNSI scores were statistically significant $(P=0.034)$. Variances in anxiety symptomatology were statistically significant $(P=0.032)$. However, at the post hoc analysis we observed that anxiety scores between patients with problem-focused coping was significantly different compared to emotion-, social support-, and avoidance-focused coping. The post hoc analysis revealed that among the latter three subgroups, the differences were not significant. Despite the variance in average BMI among the four subgroups not being statistically significant, it was observed at the post hoc analysis that patients who used social support-focused coping had significantly lower average BMI $\left(30.4 \mathrm{~kg} / \mathrm{m}^{2}\right)$ compared to any of the other subgroups.

\section{Discussion}

This study's results point to an association between the dominant coping style used by the patient and the quality of DRSCA measures. It was observed that patients with emotion-focused and social support-focused coping tended to implement more appropriate DRSCA to manage their disease. On analysis, it can be observed that the differences cannot be related to patient's age, diabetes duration, or differences in other psychological dimensions measured, since no differences in respect to these parameters were observed among the coping groups.

Physical exercise was a measure that was not properly implemented in patients who had problem-focused and especially avoidance-focused coping. Since physical exercise is a measure of paramount importance in reducing insulin resistance associated with T2DM, it becomes clear 
Table 4 Association between coping style and other diabetes-related parameters

\begin{tabular}{|c|c|c|c|c|c|}
\hline Parameter & Problem & Emotion & Social support & Avoidance & $P$-value \\
\hline Age (years) ${ }^{a}$ & $60(14)$ & $62(12)$ & $57(27)$ & $57(19)$ & 0.274 \\
\hline Diabetes duration (years) ${ }^{\mathrm{a}}$ & $12(10)$ & $12(7)$ & $9(9)$ & $13(10)$ & 0.168 \\
\hline BMI $\left(\mathrm{kg} / \mathrm{m}^{2}\right)^{\mathrm{b}}$ & $31.4 \pm 4.9$ & $32.9 \pm 6.1$ & $30.4 \pm 4.3$ & $32.8 \pm 3.7$ & 0.178 \\
\hline $\mathrm{SBP}(\mathrm{mmHg})^{\mathrm{b}}$ & $136.7 \pm 15.6$ & $138.1 \pm 18.7$ & $131.1 \pm 13.7$ & $134.2 \pm 19.9$ & 0.327 \\
\hline $\mathrm{DBP}(\mathrm{mmHg})^{\mathrm{b}}$ & $81.0 \pm 12.7$ & $86.5 \pm 9.6$ & $76.9 \pm 8$ & $79.2 \pm 7.9$ & $0.044 *$ \\
\hline eGFR $(\mathrm{mL} / \mathrm{min})^{\mathrm{b}}$ & $67.1 \pm 14.4$ & $68.5 \pm 14.2$ & $65.1 \pm 21.2$ & $71.8 \pm 20.6$ & 0.672 \\
\hline MNSI score ${ }^{a}$ & II.5 (4) & $6.5(7)$ & $10(6)$ & $8.5(9)$ & $0.034 *$ \\
\hline PHQ-9 score ${ }^{a}$ & II (9) & II (II) & $12(5)$ & II (9) & 0.786 \\
\hline GAD-7 score ${ }^{a}$ & $12(6)$ & $8(10)$ & $9(9)$ & $9(\mathrm{II})$ & $0.032 *$ \\
\hline Dyslipidemia (\%) & $37(82.2)$ & $19(51.4)$ & $21(65.6)$ & $6(50)$ & $0.017 *$ \\
\hline Optimal glycemic control reached (\%) & $9(20)$ & II (29.7) & 0 & $3(25)$ & $0.012 *$ \\
\hline
\end{tabular}

Notes: ${ }^{*} \alpha<0.05$. ${ }^{a}$ Numeric variables without Gaussian distribution. Results presented as median and interquartile range. $P$-values calculated using Kruskal-Wallis test. ${ }^{b}$ Numeric variables with Gaussian distribution. Results presented as mean \pm standard deviation. $P$-values calculated using analysis of variance. ${ }^{c} D$ ichotomous variables. Results presented as $n(\%)$. P-values calculated using $\chi^{2}$ test.

Abbreviations: BMI, body mass index; SBP, systolic blood pressure; DBP, diastolic BP; eGFR, estimated glomerular filtration rate; MNSI, Michigan Neuropathy Screening Instrument; PHQ, Patient Health Questionnaire; GAD, Generalized Anxiety Disorder (questionnaire).

that the importance of this measure should be emphasized to patients with this type of coping to obtain improved glycemic control.

An interesting aspect was that despite similar diabetes duration and patient age, regarding neuropathy, a frequently associated DM complication, the severity of the MNSI score was significantly different among dominant coping groups. This raises the question of whether the presence of this complication, which was demonstrated to decrease quality of life in affected patients, influenced the individual coping style or whether different coping styles are associated with increased prevalence of neuropathy, mediated by some mechanisms that may lead to the occurrence of neuropathy. ${ }^{14}$

The main strength of this study is represented by the fact that to our knowledge, it is the first to explore the possible link between two psychologically related components linked to diabetes: quality of DRSCA measures implemented by patients and the dominant coping style used by patients to deal with difficult and stressful situations. The main limits of the study are related to its cross-sectional character: there was a lack of information regarding DM-related parameters from the past; however, these were not related to the main objective of the study, and could only have influenced the development of several DM complications that were only secondary to the study.

Diabetes care is of paramount importance in Romanian public health. As in other countries, in Romania T2DM is a major public health issue, being a disease with high prevalence (estimated at approximately 9\%), continuously increasing incidence, and with a major impact on morbidity and mortality. Therefore, we can conclude that the results presented in this paper, which can be partially applied to the
Romanian T2DM population, may have a significant impact on these patients' treatment paradigm.

Regarding further research, the study team aims to expand this research on a group of patients with T1DM and other cohorts of patients with DM, like the hemodialyzed ones that were already demonstrated to have a series of metabolic imbalances, possibly related to lack of adherence to prescribed diet. ${ }^{15}$ Another cohort of patients with chronic diseases in which we aim to expand are patients with thyroid diseases: autoimmune thyroid diseases and thyroid cancers. ${ }^{16,17}$

\section{Conclusion}

Dominant coping mechanisms are having a significant impact on the quality of DRSCA measures implemented by patients to manage DM. Patients with adaptive coping styles (emotion-focused and social support-focused) tend to have significantly increased adherence to DRSCA, while patients with maladaptive coping strategies are less interested in managing their DM. Since the quality of DRSCA was demonstrated to be associated with improved DM prognosis, we can indirectly conclude that patients with adaptive coping styles are more likely to have improved overall DM prognosis.

\section{Disclosure}

The authors report no conflicts of interest in this work.

\section{References}

1. International Diabetes Federation. IDF Diabetes Atlas. 7th ed. Brussels: IDF; 2015.

2. Serban V, Brink S, Timar B, et al. An increasing incidence of type 1 diabetes mellitus in Romanian children aged 0 to 17 years. $J$ Pediatr Endocrinol Metab. 2015;28(3-4):293-298. 
3. Timar R, Velea I, Timar B, et al. Factors influencing the quality of life perception in patients with type 2 diabetes mellitus. Patient Prefer Adherence. 2016;10:2471-2477.

4. American Diabetes Association. Comprehensive medical evaluation and assessment of comorbidities. 2017. Diabetes Care. 2017;40 Suppl 1: S25-S32.

5. Habra ME, Linden W, Anderson JC, Weinberg J. Type D personality is related to cardiovascular and neuroendocrine reactivity to acute stress. J Psychosom Res. 2003;55(3):235-245.

6. Papava I, Oancea C, Enatescu VR, et al. The impact of coping on the somatic and mental status of patients with COPD: a cross-sectional study. Int J Chron Obstruct Pulmon Dis. 2016;11:1343-1351.

7. World Health Organization. Physical Status: The Use and Interpretation of Anthropometry. Geneva: WHO; 1995.

8. Stevens PE, Levin A. Evaluation and management of chronic kidney disease - synopsis of the Kidney Disease: Improving Global Outcomes 2012 clinical practice guideline. Ann Intern Med. 2013;158(11): 825-830.

9. Herman WH, Pop-Busui R, Braffett BH, et al. Use of the Michigan Neuropathy Screening Instrument as a measure of distal symmetrical peripheral neuropathy in type 1 diabetes: results from the Diabetes Control and Complications Trial/Epidemiology of Diabetes Interventions and Complications. Diabet Med. 2012;29(7):937-944.

10. Löwe B, Unutzer J, Callahan CM, Perkins AJ, Kroenke K. Monitoring depression treatment outcomes with the Patient Health Questionnaire-9. Med Care. 2004;42(12):1194-1201.
11. Spitzer RL, Kroenke K, Williams JBW, Löwe B. A brief measure for assessing generalized anxiety disorder: the GAD-7. Arch Intern Med. 2006;166(10):1092-1097.

12. Toobert D, Hampson S, Glasgow RE. The summary of diabetes self-care activities measure: results from 7 studies and a revised scale. Diabetes Care. 2000;23(7):943-950.

13. Craşovan DI, Sava FA. Translation, adaptation, and validation on Romanian population of Cope Questionnaire for coping mechanisms analysis. Cogn Brain Behav. 2013;17(1):61-76.

14. Timar B, Timar R, Schiller A, et al. Impact of neuropathy on the adherence to diabetes-related self-care activities: a cross-sectional study. Patient Prefer Adherence. 2016;10:1169-1175.

15. Schiller A, Gadalean F, Schiller O, et al. Vitamin D deficiency prognostic marker or mortality risk factor in end stage renal disease patients with diabetes mellitus treated with hemodialysis - a prospective multicenter study. PLoS One. 2015;10(5):e0126586.

16. Stoian D, Timar B, Derban M, et al. Thyroid Imaging Reporting and Data System (TI-RADS): the impact of quantitative strain elastography for better stratification of cancer risks. Med Ultrason. 2015;17(3):327-332.

17. Vlad M, Golu I, Bota S, et al. Real-time shear wave elastography may predict autoimmune thyroid disease. Wien Klin Wochenschr. 2015; 127(9-10):330-336
Patient Preference and Adherence

\section{Publish your work in this journal}

Patient Preference and Adherence is an international, peer-reviewed, open access journal that focuses on the growing importance of patient preference and adherence throughout the therapeutic continuum. Patient satisfaction, acceptability, quality of life, compliance, persistence and their role in developing new therapeutic modalities and compounds to optimize

\section{Dovepress}

clinical outcomes for existing disease states are major areas of interest for the journal. This journal has been accepted for indexing on PubMed Central. The manuscript management system is completely online and includes a very quick and fair peer-review system, which is all easy to use. Visit http://www. dovepress.com/testimonials.php to read real quotes from published authors. 